\title{
Gestão da qualidade e utilização do Método de Ishikawa na diminuição do Tempo de Permanência dos Veículos - TPV Inbound: estudo de caso
}

\section{Quality management utilization Method in Ishikawa Decreased Dwell Time Vehicle - POS Inbound: case study}

\author{
${ }^{1}$ Eriane Fialho Carvalho \\ ${ }^{1}$ Alexander Santos \\ ${ }^{1}$ Avanil Silva \\ 'Edson Correa \\ 'Rodolfo Martins \\ 1 Tássia Santos \\ 1 Thalita Reis
}

1 Universidade Estadual Paulista - Júlio de Mesquita Filho -UNESP, Guaratinguetá -SP.

\section{Resumo}

O objetivo deste trabalho é correlacionar uma das ferramentas da qualidade em um case real, no qual foi utilizada a ferramenta Ishikawa como príncipio básico para solucionar o problema detectado. 0 case analisado consiste na redução do tempo médio que os veículos permanecem dentro da usina, aguardando o devido carregamento, uma vez que se entende por qualidade $o$ atendimento das necessidades do cliente continuamente, baseando-se na prevenção de aspectos relativos a não qualidade, tais como: erros, defeitos na realização de serviços e produção de bens, erro na compra de produtos, serviço desnecessário, produtos inseguros, falta de segurança nas condições de trabalho, tempo desperdiçado, demoras e falhas. Sendo assim, o tempo ocioso do veículo no interior da usina deixa-os expostos a riscos quanto à segurança das pessoas, ao relacionamento entre motoristas e demais colaboradores envolvidos nas execuções, a custos, à produtividade e, consequentemente, atraso da entrega do produto ao cliente.

\section{Palavras-chave}

Gestão da Qualidade; ferramenta de qualidade; ishikawa; custo.

\begin{abstract}
The objective of this work is to relate one of quality tools in a real case, in which we used the Ishikawa tool as a basic principle to solve the problem detected. The analyzed case is to reduce the average time that the vehicles remain within the plant, waiting for the proper loading, since what is meant by quality meet the customer needs continuously, based on the prevention aspects of not quality, such as errors, defects in the performance of services and production of goods, error in the purchase of products, services unnecessary, unsafe products, unsafe working conditions, wasted time, delays and failures. Thus, the downtime of the vehicle within the plant leaves them exposed to risks to the safety of persons, the relationship between drivers and other employees involved in executions, costs, productivity and consequently delay the delivery of the product to client.
\end{abstract}

\section{Keywords}

Quality Management; quality tool; Ishikawa; cost.

\section{Como você deve citar?}

CARVALHO, Eriane Fialho. Gestão da qualidade e utilização do Método de Ishikawa na diminuição do Tempo de Permanência dos Veículos - TPV Inbound: estudo de caso. Cadernos UniFOA, Volta Redonda, n. 28, p. 31-38, ago. 2015. 
Gestão da qualidade e utilização do Método de Ishikawa na diminuição do Tempo de Permanência dos Veículos - TPV Inbound: estudo de caso

\section{INTRODUÇÃO}

Qualidade é algo que, atualmente, é parte integrante de nossas vidas, abrangendo desde aspectos particulares e individuais até os de serviço e sociedade. Qualidade não é somente um produto ou um serviço que adquirimos e usamos em casa, é também a forma como estamos, como nos comportamos, como convivemos, social e profissionalmente na vida.

Qualquer empresa, atualmente, só sobrevive se produzir ou vender atendendo expectativas dos clientes, devendo ser essa a maior preocupação da alta gerência: a total satisfação das necessidades das pessoas afetadas pela sua existência.

Desde que se iniciou a preocupação pela qualidade, diversos "métodos de melhoria" passaram a ser aplicados nos processos, cada um com seu procedimento definido, fazendo uso de ferramentas clássicas de qualidade.

Os programas de qualidade focam em eficiência, ou seja, redução das não conformidades e dos custos de garantia. Empresas que têm utilizado seis sigma divulgam cifras milionárias de ganhos obtidos com sua implementação. Isso ocorre devido à harmoniosa integração do gerenciamento por processo e por diretrizes, focando sempre nos clientes, nos processos críticos e nos resultados.

O método utilizado para a análise dos problemas foi baseado nas ferramentas de Lean - Seis sigma, optando-se pelo diagrama de causa e efeito (Ishikawa) para a redução do tempo de permanência dos veículos dentro de uma usina.

TPV é a medida do tempo médio que os veículos permanecem dentro da usina, aguardando carregamento. Isso impacta em segurança das pessoas (colaboradores de logística e motoristas prestadores de serviço), clima, custos e produtividade.

A partir dessa premissa, apresentaremos uma parte teórica que tem como referência artigos, nos quais foram abordados assuntos como: Seis Sigma, ferramentas de qualidade e diagrama de causa e efeito (Ishikawa). A partir desses artigos foram feitas análises e correlacionadas com o case estudado.

Na seção 3, foi descrito o desenvolvimento da aplicação da ferramenta para a solução do problema diagnosticado. A seção 4 discute os resultados obtidos após a inserção da ferramenta e, finalmente, na seção 5 , foi concluído o estudo.

\section{REFERÊNCIAL TEÓRICO}

De acordo com Reis e Mañas (1994), "qualidade é a totalidade dos aspectos e características de um produto ou serviço relacionada a sua capacidade de satisfazer as necessidades declaradas ou implícitas".

Mello et al. (2002) diz que o escopo do sistema de gestão da qualidade deveria ser baseado na natureza dos produtos e realização dos processos da organização, nos resultados das análises de risco, nas considerações comerciais e nos requisitos contratuais e regulamentares.

O sistema Seis Sigma abrange todas as atividades de uma empresa, propondo ações de melhoria para todos os seus setores. 
O método introduzido por W. Edwards Deming, o Ciclo PDCA (Planejar, executar, verificar, agir) é um dos exemplos mais populares de metodologia que tem sido utilizado para a melhoria dos processos.

O modelo Maic (Medir, Analisar, Melhorar, Controlar) foi desenvolvido, inicialmente, na Motorola como uma evolução do ciclo PDCA e, depois, adotado pela GE como DMAIC, em que o D significa a fase "definir". Esse método passou a ser a base operacional da ruptura Seis Sigma para essas empresas, sendo fundamental para o sucesso que alcançaram.

Apesar de colocados em termos diferentes, o método está centrado na identificação dos problemas-base para a seleção dos projetos a serem executados; na coleta de dados de forma honesta, que leva a conhecer o desempenho do processo atual; na determinação das causas dos problemas, que leva à análise das causas; na formulação de ações de melhoria, que leva à melhoria do processo; na consolidação e manutenção das melhorias conseguidas, que leva a manter o processo sob controle. (ROTONDARO, 2002).

Nos projetos de melhoria Seis Sigma, o resultado do processo é um problema que se quer eliminar. Sendo assim o diagrama de causa e efeito é utilizado para fazer um levantamento e uma apresentação visual de suas possíveis causas e seu relacionamento com o problema.

Seis Sigma é uma ferramenta tática de valor significativo para alcançar a excelência operacional. A seleção de projetos de decisão, no âmbito de uma restrição de recursos, é a fase inicial de implementação de uma intervenção de Seis Sigma (YANG, 2009).

Sobre o diagrama de causa e efeito, pode-se afirmar:

- É conhecido por espinha de peixe ou Ishikawa. Representa a relação entre um efeito (problema) e as suas potenciais causas;

- Ordena e relaciona fatores que afetam um processo, enquanto dados poucos quantificáveis estão disponíveis;

- Ajuda a determinar a causa real de problema vs. um sintoma. (COSTA, 2012).

Esse diagrama tem por função expandir o leque de informações sobre o problema e aumentar a probabilidade de uma correta identificação das principais causas para que estas possam ser atacadas. Essa abertura deve ser a mais ampla possível e ser desenvolvida com a participação de um grupo de colaboradores que tenham grande envolvimento e pleno conhecimento sobre o processo e o problema ocorrente.

\subsection{Impactos do TPV dentro da usina:}

- Segurança das pessoas: quanto maior o tempo em que os motoristas permanecem no pátio ou no interior da usina, maior a exposição aos riscos, devido a fatores como excesso de movimentações, ansiedade, distúrbios provocados pela falta de perspectiva de carregamento e, até mesmo, risco de realização de manobras ou operações irregulares para agilizar o carregamento.

- Clima: o relacionamento entre motoristas e colaboradores da logística e das células operacionais é prejudicado pelo excesso de tempo de permanência dos motoristas na usina. 0 ambiente de trabalho fica comprometido, além de acumular vários caminhões no pátio ao mesmo tempo com excesso de movimentações, tanto no interior da usina como no lado de fora (pátio). 
- Custos: quanto maior o tempo de permanência dos veículos na usina, aguardando o carregamento, maiores serão os custos de frete, pois os fornecedores de serviços logísticos (transportadoras) incluem o tempo de espera no valor do frete, devido às estatísticas que levantam de cada empresa. 0 tempo que os veículos esperam, às vezes, chega a ser maior que a própria locomoção (rota) de ida e volta entre carga e descarga. Com a redução do TPV, a empresa pode pleitear redução dos preços de movimentação e deixar de pagar multas por atraso no carregamento.

- Produtividade: a perda de produtividade se dá devido ao fato de o mesmo transporte aguardar um tempo acima do esperado para carregar, ter que retornar para outro carregamento e, no final do mês, o número de carretos que são realizados é menor que o planejado.

- Outros: atraso na entrega e perda de embarque.

\section{DESENVOLVIMENTO DA APLICAÇÃO}

Mudanças relevantes:

- Mudança de leiaute: ação tomada para facilitar a entrada dos caminhões na portaria da usina. Atualmente, não existe fila para entrar na usina.

- Medição de leiaute - oscilação: ação tomada para aperfeiçoar os leiautes para carregamento e descarga.

- Criação de rotina: com a definição de tempos, criou-se um padrão de movimentação, no qual todos os operadores envolvidos participarão da criação e garantem a execução.

- Estabilização de processo: através do avião da qualidade, foi possível identificar as tarefas críticas, com medição dos desvios, tratamento das falhas imediatas e auditoria, garantindo a estabilização.

- Definição de especialistas - características - padrão de localização fixa: pessoas por postos de descarregamento e carregamento, treinadas e com autonomia para tomada de decisão.

- Pavimentação: asfaltamento de todas as vias de trânsito de caminhões.

- Iluminação: troca da iluminação das vias de trânsito de caminhões, garantindo a segurança durante todo o transporte.

- Conscientização: após implementação, a divulgação das ações foi feita para todas as pessoas da usina, de maneira presencial pelos gestores da planta, com envolvimento total da liderança .

- Gestão de estoques: monitoramento dos caminhões, desde a entrada até a saída, com controle exato do que foi descarregado fisicamente.

- Ciência para as transportadoras: reuniões sistemáticas, envolvendo todas as transportadoras no mesmo objetivo de reduzir o TPV. 


\section{RESULTADOS}

\subsection{Análise do TPV por frete}

Figura 1 - Análise do TPV tara-nota

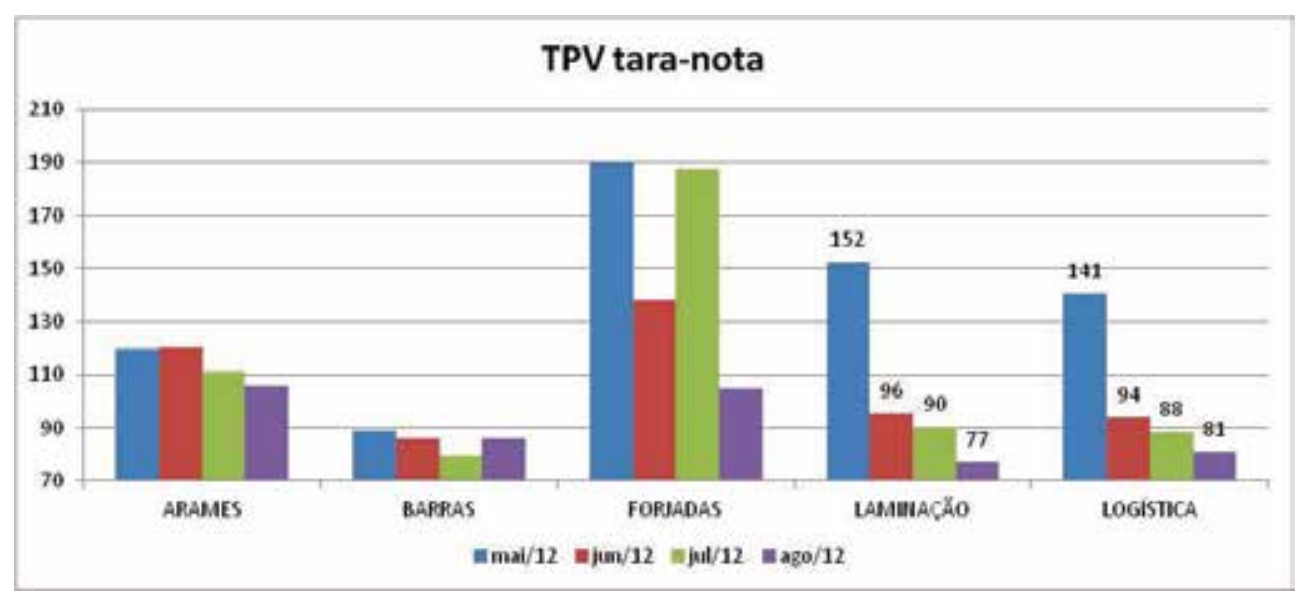

Queda no TPV TR-NF

LAMINADOS LOGÍSTICA

\begin{tabular}{|l|r|r|}
\hline mai =>jun & 57 & 47 \\
\hline jun=>jul & 5 & 6 \\
\hline jul=>ago & 13 & 7 \\
\hline total TRI & 75 & 60 \\
\hline
\end{tabular}

Figura 2 - Análise do TPV abertura saída.

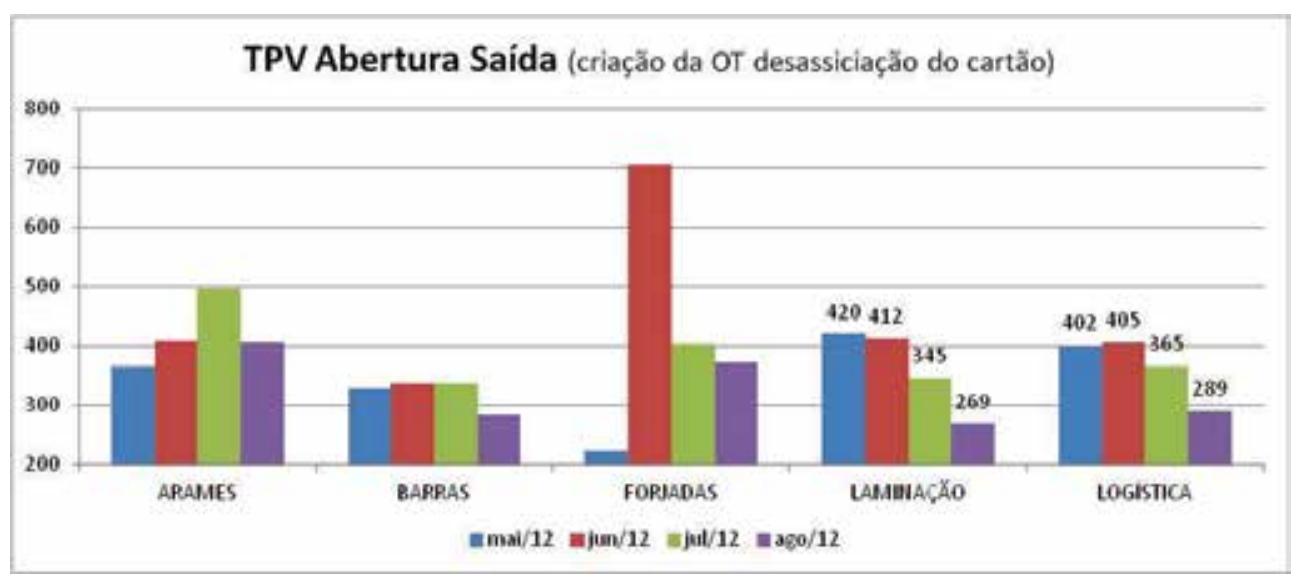

\begin{tabular}{|l|r|r|}
\cline { 2 - 3 } \multicolumn{1}{c|}{} & \multicolumn{2}{c|}{ Queda no TPV Abert Saída } \\
\cline { 2 - 3 } \multicolumn{1}{c|}{} & LAMINADOS & LOGÍSTICA \\
\hline mai=>jun & 7 & -3 \\
\hline jun=>jul & 67 & 40 \\
\hline jul=>ago & 76 & 76 \\
\hline total TRI & 151 & 112 \\
\hline
\end{tabular}


Gestão da qualidade e utilização do Método de Ishikawa na diminuição do Tempo de Permanência dos Veículos - TPV Inbound: estudo de caso

As figuras 1 e 2 mostram as análises do tempo de permanência dos veículos por seção. Foram analisadas as seguintes seções: arames, barras, forjadas, laminação e logísticas nos meses de maio, junho, julho e agosto de 2012.

Foi possível observar a diminuição significativa do TPV em todas as seções, após a aplicação do estudo, feito com o auxílio do diagrama de causa e efeito.

\subsection{Análise do TPV por transportadora}

Figura 3 - Análise do TPV abertura saída - laminados.

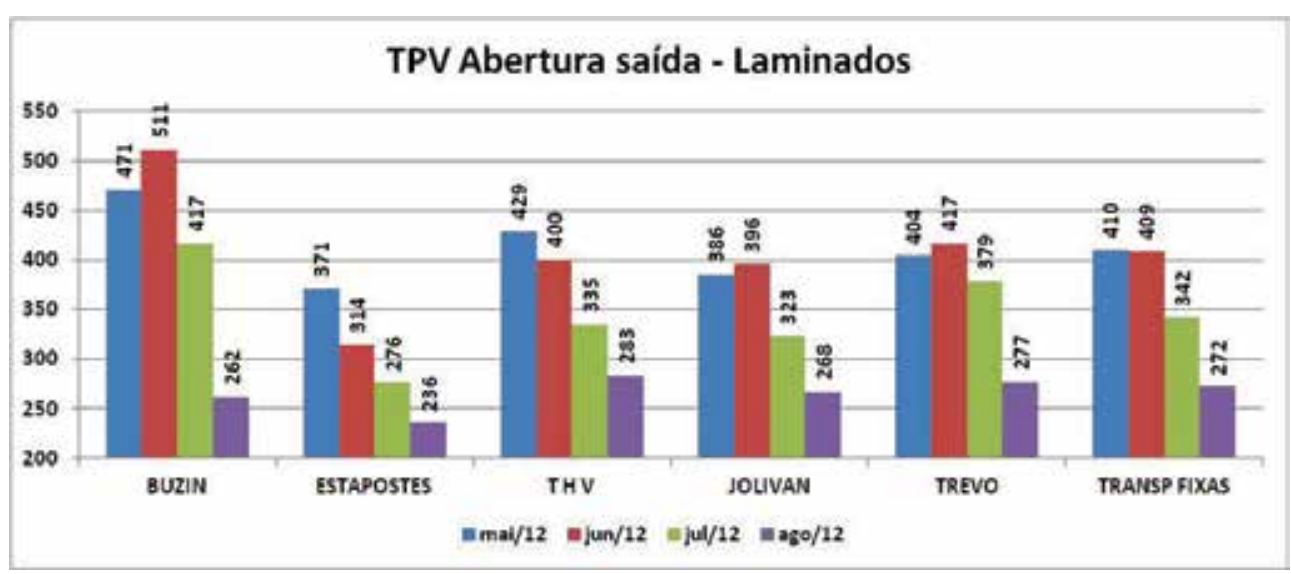

Figura 4 - Análise do TPV entrada saída.

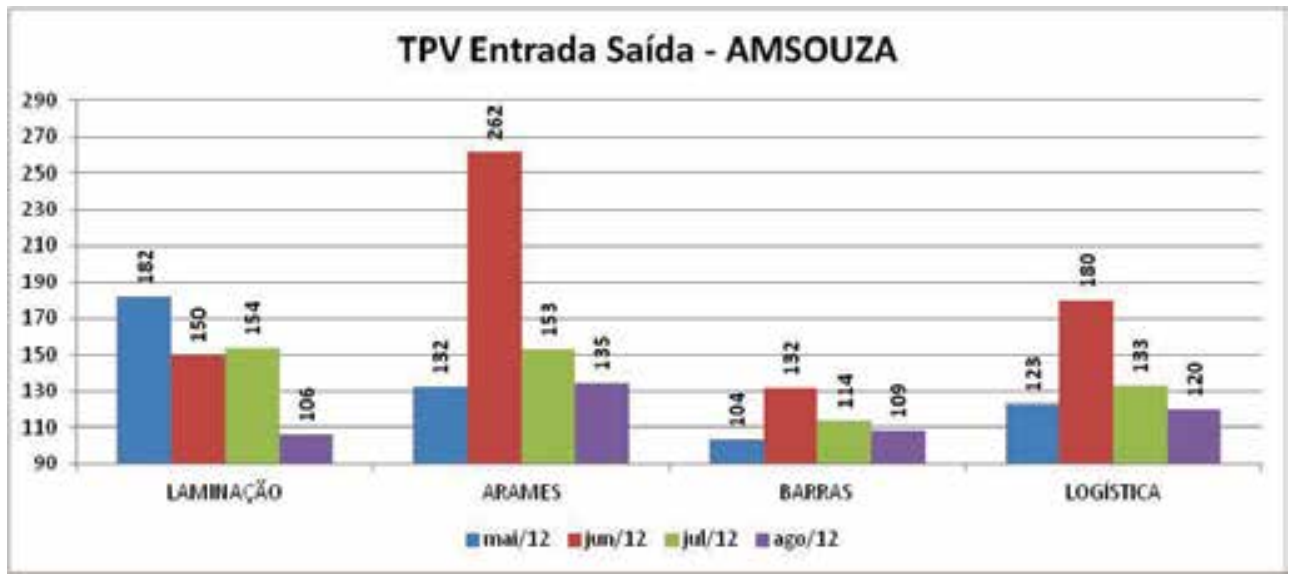

As figuras 3 e 4 mostram as análises do tempo de permanência dos veículos em duas transportadoras, nos meses de maio, junho, julho e agosto de 2012.

Foi possível observar a diminuição significativa do TPV em todas as seções, após a aplicação do estudo feito com o auxílio do diagrama de causa e efeito, independente da transportadora utilizada. 


\subsection{TPV x Faturamento}

Figura 5 - Análise do TPV x Faturamento.

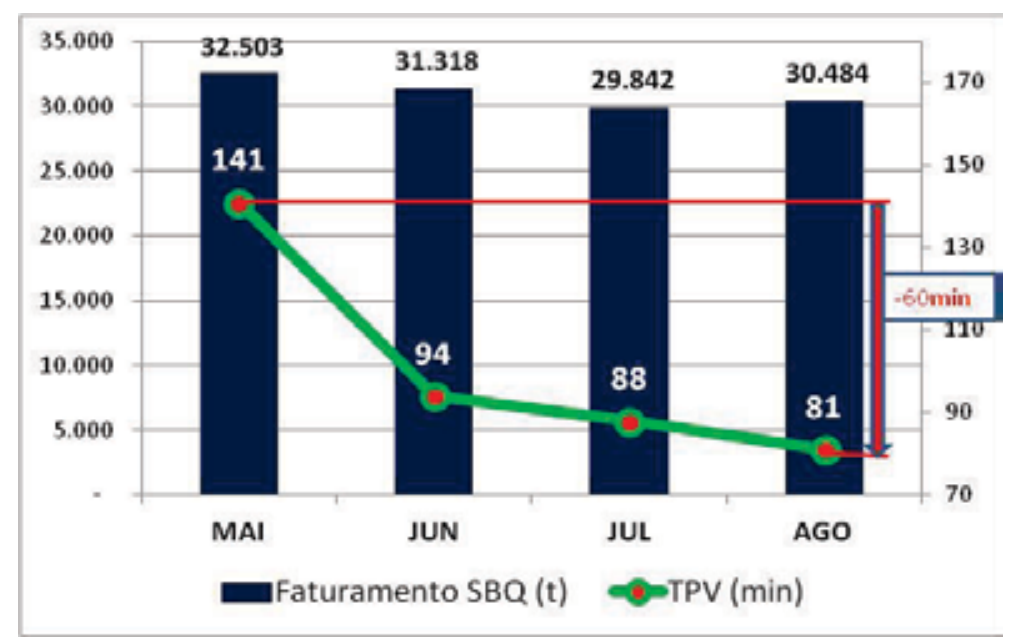

Na figura 5, foi análisado o impacto que a redução do TPV causou no faturamento da empresa.

Foi possível observar que a redução do TPV impactou de forma positivada no faturamento e que, mesmo com um menor TPV, obteve-se maior faturamento.

\subsection{Criação de Rotinas e Postos de Trabalho}

Figura 6 - Antes e depois da criação de rotinas e postos de trabalho.

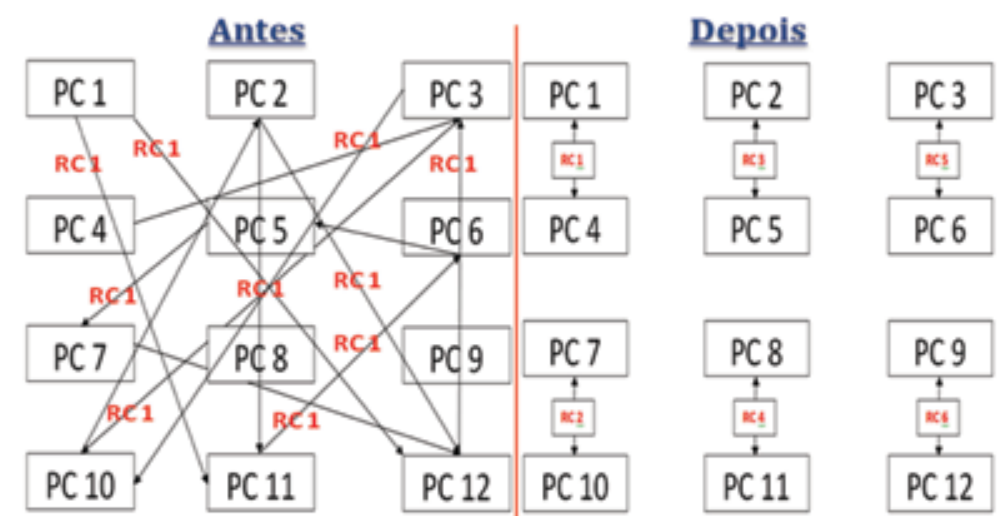

A figura 6 mostra a rotina dos postos de trabalho antes e depois da aplicação deste estudo.

Percebeu-se, com clareza, uma melhoria na rotina e nos postos de trabalho após a aplicação. 
Gestão da qualidade e utilização do Método de Ishikawa na diminuição do Tempo de Permanência dos Veículos - TPV Inbound: estudo de caso

\section{CONCLUSÃO}

Analisando os resultados, foi possível perceber que, quanto menor o TPV, maior é o faturamento da empresa.

Percebeu-se, também, que após a aplicação deste estudo, houve redução do TPV em todos os setores analisados.

Quando se fez a análise por transportadoras, percebeu-se que também houve uma redução do TPV, independente de qual era a empresa de transporte.

Após a aplicação de todos esses conceitos, percebeu-se uma redução de 6,61\% do custo da transportadora e uma redução de $28 \%$ do TPV de maio/2012 para agosto/2012.

\section{REFERÊNCIAS}

BÜYÜKÖZKAN, G.; ÖZTÜRKCAN, D. An integrated analytic approach for Six Sigma project selection. Expert Systems with Applications. 5835-5847. Turkey. 2010. Disponível em:< http://wokinfo.com/>. Acesso em: 21 maio 2013.

CHAKRAVORTY, S. S. Six Sigma programs: An implementation model. International journal of production economics. 1-16. USA. 2009. Disponível em:< http://wokinfo.com/>. Acesso em: 21 maio 2013.

COSTA, G. H. Apostila do curso de formação de Green Belt-6 sigma. RL \& Associados. 2012.

KUMAR, U. D. et al. On the optimal selection of process alternatives in a Six Sigma implementation. International journal of production economics. 456-467. USA. 2008. Disponível em:< http://wokinfo. com/>. Acesso em: 21 maio 2013.

MELLO, C. H. P et al. ISO 9001:2000: sistema de gestão da qualidade para operações de produção e serviços. São Paulo: Atlas, 2002.

REIS, L. F. S. D.; MAÑAS, A. V. ISO 9000: um caminho para a qualidade total. São Paulo: Érica, 1994.

ROTONDARO, R. G. et al. Seis sigma: estratégia gerencial para a melhoria de processos, Produtos e Serviços. São Paulo: Atlas, 2002.

SU, C.; CHOU, C. A systematic methodoly for the creation of Six Sigma projects: A case study of semiconductor foundry. Expert Systems with Applications. 2693-2703. Taiwan. 2008. Disponível em:< http://wokinfo.com/>. Acesso em: 21 maio 2013.

YANG, T.; HSIEH, C. Six-Sigma project selection using national quality award criteria and Delphi fuzzy multiple criteria decision-making method. Systems with Applications. 7594-7603. Taiwan. 2009. Disponível em:< http://wokinfo.com/>. Acesso em: 21 maio 2013.

ZU, X.; ROBBINS, T. L.; FREDENDALL, L. D. Mapping the critical links between organizational culture and TQM/SIX Sigma practices. International journal of production economics. 86-106. USA. 2010. Disponível em:< http://wokinfo.com/>. Acesso em: 21 maio 2013. 